\title{
Kinematical structure of the Magellanic System
}

\author{
Roeland P. van der Marel $^{1}$, Nitya Kallivayalil ${ }^{2}$ and Gurtina Besla ${ }^{3}$ \\ ${ }^{1}$ Space Telescope Science Institute, 3700 San Martin Drive, Baltimore, MD 21218, USA \\ ${ }^{2}$ MIT, Kavli Inst. for Astrophysics \& Space Research, 70 Vassar Street, \\ Cambridge, MA 02139, USA \\ ${ }^{3}$ Harvard-Smithsonian Center for Astrophysics, 60 Garden Street, Cambridge, MA 02138, USA
}

\begin{abstract}
We review our understanding of the kinematics of the LMC and the SMC, and their orbit around the Milky Way. The line-of-sight velocity fields of both the LMC and SMC have been mapped with high accuracy using thousands of discrete traces, as well as H I gas. The LMC is a rotating disk for which the viewing angles have been well established using various methods. The disk is elliptical in its disk plane. The disk thickness varies depending on the tracer population, with $V / \sigma$ ranging from $\sim 2-10$ from the oldest to the youngest population. For the SMC, the old stellar population resides in a spheroidal distribution with considerable line-of-sight depth and low $V / \sigma$. Young stars and H I gas reside in a more irregular rotating disk. Mass estimates based on the kinematics indicate that each Cloud is embedded in a dark halo. Proper motion measurements with HST show that both galaxies move significantly more rapidly around the Milky Way than previously believed. This indicates that for a canonical $10^{12}$ $\mathrm{M}_{\odot}$ Milky Way the Clouds are only passing by us for the first time. Although a higher Milky Way mass yields a bound orbit, this orbit is still very different from what has been previously assumed in models of the Magellanic Stream. Hence, much of our understanding of the history of the Magellanic System and the formation of the Magellanic Stream may need to be revised. The accuracy of the proper motion data is insufficient to say whether or not the LMC and SMC are bound to each other, but bound orbits do exist within the proper motion error ellipse.
\end{abstract}

Keywords. stellar dynamics, celestial mechanics, astrometry, ISM: kinematics and dynamics, Galaxy: halo, galaxies: kinematics and dynamics, Magellanic Clouds, galaxies: structure, dark matter

\section{Introduction}

The Magellanic Clouds are two of the closest galaxies to the Milky Way, with the Large Magellanic Cloud (LMC) at a distance of $\sim 50 \mathrm{kpc}$ and the Small Magellanic Cloud $(\mathrm{SMC})$ at $\sim 62 \mathrm{kpc}$. Because of their proximity, they are two of the best-studied galaxies in the Universe. As such, they are a benchmark for studies on various topics, including stellar populations and the interstellar medium, microlensing by dark objects, and the cosmological distance scale. As nearby companions of the Milky Way with significant signs of mutual interaction, they have also been taken as examples of hierarchical structure formation in the Universe. For all these applications it is important to have an understanding of the kinematics of the LMC and the SMC, as well the kinematics (i.e., orbit) of their center of mass with respect to the Milky Way and with respect to each other. These topics form the subject of the present review. Other related topics, such as the more general aspects of the structure of the LMC and SMC, the nature of the LMC bar, the possible presence of fore- or background populations, and the large radii extent of the Clouds are not discussed here. The nature, origin, and models of the Magellanic 
Stream are touched upon only briefly. All these topics are reviewed in other papers in this volume by, e.g., Majewski, Besla, Bekki, and others.

\section{LMC kinematics}

Kinematical observations for the LMC have been obtained for many tracers. The kinematics of gas in the LMC has been studied primarily using H I (e.g., Kim et al. 1998; Olsen \& Massey 2007). Discrete LMC tracers which have been studied kinematically include star clusters (e.g., Schommer et al. 1992; Grocholski et al. 2006), planetary nebulae (Meatheringham et al. 1988), H II regions (Feitzinger, Schmidt-Kaler \& Isserstedt 1977), red supergiants (Olsen \& Massey 2007), red giant branch (RGB) stars (Zhao et al. 2003; Cole et al. 2005), carbon stars (e.g., van der Marel et al. 2002; Olsen \& Massey 2007) and RR Lyrae stars (Minniti et al. 2003; Borissova et al. 2006). For the majority of tracers, the line-of-sight velocity dispersion is at least a factor $\sim 2$ smaller than their rotation velocity. This implies that on the whole the LMC is a (kinematically cold) disk system.

\subsection{General expressions}

To understand the kinematics of an LMC tracer population it is necessary to have a general model for the line-of-sight velocity field that can be fit to the data. All studies thus far have been based on the assumption that the mean streaming (i.e. the rotation) in the disk plane can be approximated to be circular. However, even with this simplifying assumption it is not straightforward to model the kinematics of the LMC. Its main body spans more than $20^{\circ}$ on the sky and one therefore cannot make the usual approximation that "the sky is flat" over the area of the galaxy. Spherical trigonometry must be used, which yields the general expression (van der Marel et al. 2002; hereafter vdM02):

$$
\begin{aligned}
v_{\mathrm{los}}(\rho, \Phi)= & s V\left(R^{\prime}\right) f \sin i \cos (\Phi-\Theta)+v_{\mathrm{sys}} \cos \rho \\
& +v_{t} \sin \rho \cos \left(\Phi-\Theta_{t}\right)+D_{0}(d i / d t) \sin \rho \sin (\Phi-\Theta),
\end{aligned}
$$

with

$$
R^{\prime}=D_{0} \sin \rho / f, \quad f \equiv \frac{\cos i \cos \rho-\sin i \sin \rho \sin (\Phi-\Theta)}{\left[\cos ^{2} i \cos ^{2}(\Phi-\Theta)+\sin ^{2}(\Phi-\Theta)\right]^{1 / 2}} .
$$

Here, $v_{\text {los }}$ is the observed component of the velocity along the line of sight. The quantities $(\rho, \Phi)$ identify the position on the sky with respect to the center: $\rho$ is the angular distance and $\Phi$ is the position angle (measured from North over East). The kinematical center is at the center of mass $(\mathrm{CM})$ of the galaxy. The quantities $\left(v_{\mathrm{sys}}, v_{t}, \Theta_{t}\right)$ describe the velocity of the $\mathrm{CM}$ in an inertial frame in which the sun is at rest: $v_{\text {sys }}$ is the systemic velocity along the line of sight, $v_{t}$ is the transverse velocity, and $\Theta_{t}$ is the position angle of the transverse velocity on the sky. The angles $(i, \Theta)$ describe the direction from which the plane of the galaxy is viewed: $i$ is the inclination angle ( $i=0$ for a face-on disk), and $\Theta$ is the position angle of the line of nodes (the intersection of the galaxy plane and the sky plane). The velocity $V\left(R^{\prime}\right)$ is the rotation velocity at cylindrical radius $R^{\prime}$ in the disk plane. $D_{0}$ is the distance to the CM, and $f$ is a geometrical factor. The quantity $s= \pm 1$ is the 'spin sign' that determines in which of the two possible directions the disk rotates.

The first term in equation (2.1) corresponds to the internal rotation of the LMC. The second term is the part of the line-of-sight velocity of the CM that is seen along the line of sight, and the third term is the part of the transverse velocity of the CM that is seen along the line of sight. For a galaxy that spans a small area on the sky (very small $\rho$ ), the second term is simply $v_{\text {sys }}$ and the third term is zero. However, the LMC does not have a small angular extent and the inclusion of the third term is 
particularly important. It corresponds to a solid-body rotation component that at most radii exceeds in amplitude the contribution from the intrinsic rotation of the LMC disk. The fourth term in equation (2.1) describes the line-of-sight component due to changes in the inclination of the disk with time, as are expected due to precession and nutation of the LMC disk plane as it orbits the Milky Way (Weinberg 2000). This term also corresponds to a solid-body rotation component.

The general expression in equation (2.1) appears complicated, but it is possible to gain intuitive insight by considering some special cases. Along the line of nodes one has that $\sin (\Phi-\Theta)=0$ and $\cos (\Phi-\Theta)= \pm 1$, so that

$$
\hat{v}_{\text {los }}(\text { along })= \pm\left[v_{t c} \sin \rho-V\left(D_{0} \tan \rho\right) \sin i \cos \rho\right]
$$

Here it has been defined that $\hat{v}_{\text {los }} \equiv v_{\text {los }}-v_{\text {sys }} \cos \rho \approx v_{\text {los }}-v_{\text {sys }}$. The quantity $v_{t c} \equiv$ $v_{t} \cos \left(\Theta_{t}-\Theta\right)$ is the component of the transverse velocity vector in the plane of the sky that lies along the line of nodes; similarly, $v_{t s} \equiv v_{t} \sin \left(\Theta_{t}-\Theta\right)$ is the component perpendicular to the line of nodes. Perpendicular to the line of nodes one has that $\cos (\Phi-$ $\Theta)=0$ and $\sin (\Phi-\Theta)= \pm 1$, and therefore

$$
\hat{v}_{\text {los }}(\text { perpendicular })= \pm w_{t s} \sin \rho .
$$

Here it has been defined that $w_{t s}=v_{t s}+D_{0}(d i / d t)$. This implies that perpendicular to the line of nodes $\hat{v}_{\text {los }}$ is linearly proportional to $\sin \rho$. By contrast, along the line of nodes this is true only if $V\left(R^{\prime}\right)$ is a linear function of $R^{\prime}$. This is not expected to be the case, because galaxies do not generally have solid-body rotation curves; disk galaxies tend to have flat rotation curves, at least outside the very center. This implies that, at least in principle, both the position angle $\Theta$ of the line of nodes and the quantity $w_{t s}$ are uniquely determined by the observed velocity field: $\Theta$ is the angle along which the observed $\hat{v}_{\text {los }}$ are best fit by a linear proportionality with $\sin \rho$, and $w_{t s}$ is the proportionality constant.

\subsection{Carbon star kinematics}

vdM02 were the first to fit the velocity field expression in equation (2.1) in its most general form to a large sample of discrete LMC velocities. They modeled the data for 1041 carbon stars, obtained from the work of Kunkel, Irwin \& Demers (1997) and Hardy, Schommer \& Suntzeff (unpublished). The combined dataset samples both the inner and the outer parts of the LMC, although with a discontinuous distribution in radius and position angle. Figure 1 shows the data, with the best model fit overplotted. Overall, the model provides a good fit to the data. Olsen \& Massey (2007) recently remodeled the same carbon star data (for which they obtained a similar fit as vdM02), as well as a large sample of red supergiant stars.

\subsection{Viewing angles and ellipticity}

The LMC inclination cannot be determined kinematically, but the line-of-nodes position angle can. vdM02 obtained $\Theta=129.9^{\circ} \pm 6.0^{\circ}$ for carbon stars, whereas Olsen \& Massey (2007) obtained $\Theta=145.3^{\circ}$ for red supergiants.

A more robust way to determine the LMC viewing angles is to use geometrical considerations, rather than kinematical ones (since this avoids the assumption that the orbits are circular). For an inclined disk, one side will be closer to us than the other. Tracers on that one side will appear brighter than similar tracers on the other side. To lowest order, the difference in magnitude between a tracer at the galaxy center and a similar tracer at a position $(\rho, \Phi)$ in the disk (as defined in Section 2.1) is

$$
\mu=\left(\frac{5 \pi}{180 \ln 10}\right) \rho \tan i \sin (\Phi-\Theta),
$$



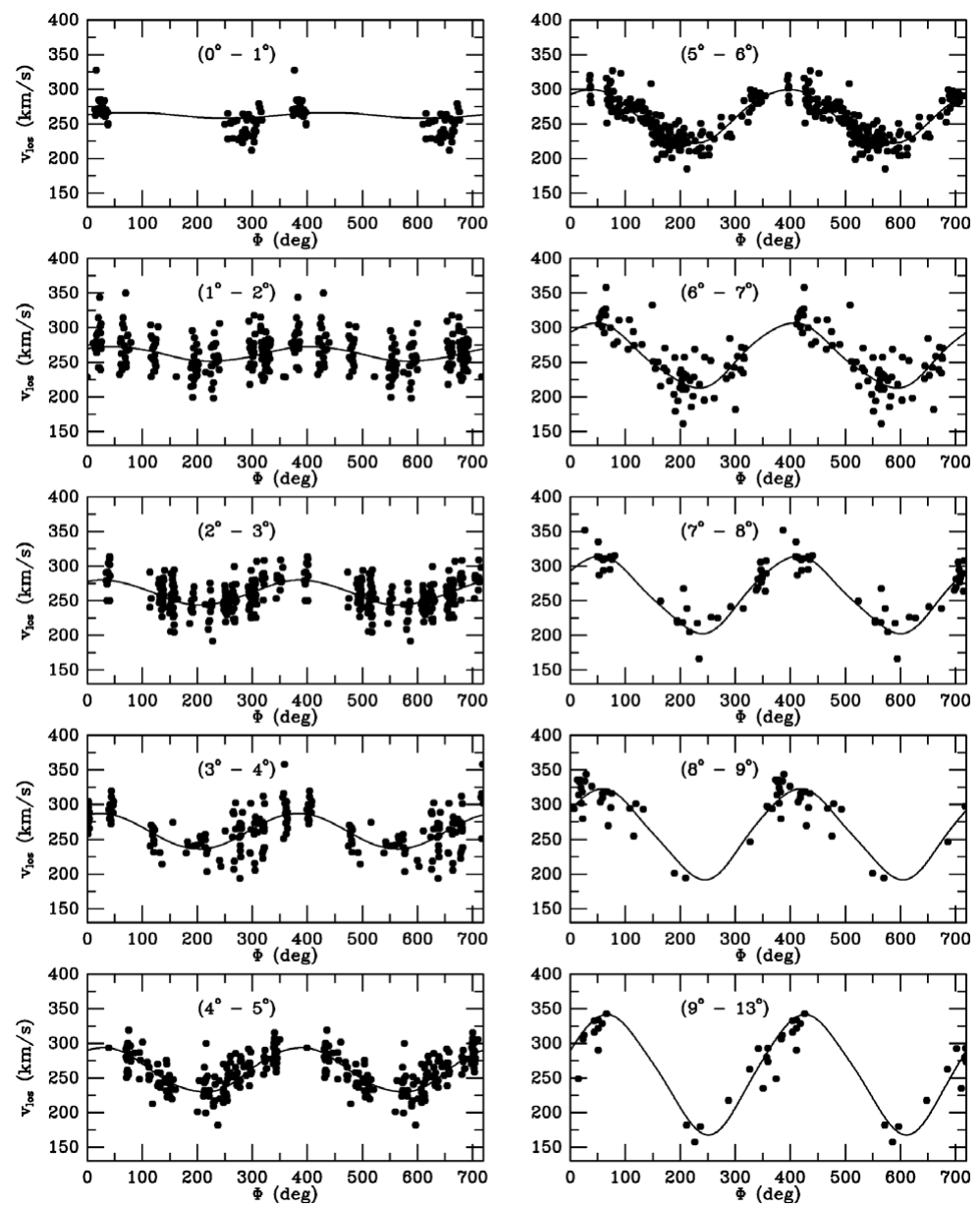

Figure 1. Carbon star line-of-sight velocity data from Kunkel et al. (1997) and Hardy et al. (unpublished), as a function of position angle $\Phi$ on the sky. The displayed range of the angle $\Phi$ is $0^{\circ}-720^{\circ}$, so each star is plotted twice. Each panel corresponds to a different range of angular distances $\rho$ from the LMC center, as indicated. The curves show the predictions of the best-fitting circularly-rotating disk model from vdM02.

where the angular distance $\rho$ is expressed in degrees. The constant in the equation is $(5 \pi) /(180 \ln 10)=0.038$ magnitudes. Hence, when following a circle on the sky around the galaxy center one expects a sinusoidal variation in the magnitudes of tracers. The amplitude and phase of the variation yield estimates of the viewing angles $(i, \Theta)$.

Van der Marel \& Cioni (2001) used a polar grid on the sky to divide the LMC area into several rings, each consisting of a number of azimuthal segments. The data from the DENIS and 2MASS surveys were used for each segment to construct near-IR colormagnitude diagrams (CMDs). For each segment both the modal magnitude of carbon stars (selected by color) and the magnitude of the RGB tip (TRGB) were determined. This revealed the expected sinusoidal variations at high significance, implying viewing angles $i=34.7^{\circ} \pm 6.2^{\circ}$ and $\Theta=122.5^{\circ} \pm 8.3^{\circ}$. There is an observed drift in the center of the LMC isophotes at large radii which is consistent with this result, when interpreted as a result of viewing perspective (van der Marel 2001). Also, Grocholski et al. (2006) found that the red clump distances to LMC star clusters are consistent with a disk-like configuration with these same viewing angles. 
The aforementioned analyses are sensitive primarily to the structure of the outer parts of the LMC. Several other studies of the viewing angles have focused mostly on the region of the bar, which samples only the central few degrees. Nikolaev et al. (2004) analyzed a sample of more than 2000 Cepheids with lightcurves from MACHO data and obtained $i=30.7^{\circ} \pm 1.1^{\circ}$ and $\Theta=151.0^{\circ} \pm 2.4^{\circ}$. Persson et al. (2004) obtained $i=27^{\circ} \pm 6^{\circ}$ and $\Theta=127^{\circ} \pm 10^{\circ}$ from a much smaller sample of 92 Cepheids. Olsen \& Salyk (2002) obtained $i=35.8^{\circ} \pm 2.4^{\circ}$ and $\Theta=145^{\circ} \pm 4^{\circ}$ from an analysis of variations in the magnitude of the red clump.

In summary, all studies agree that $i$ is approximately in the range $30^{\circ}-35^{\circ}$, whereas $\Theta$ appears to be in the range $120^{\circ}-150^{\circ}$. The variations between results from different studies may be due to a combination of systematic errors, spatial variations in the viewing angles (warps and twists of the disk plane; van der Marel \& Cioni 2001; Olsen \& Salyk 2002; Subramaniam 2003; Nikolaev et al. 2004) combined with differences in spatial sampling between studies, contamination by possible out of plane structures, and differences between different tracer populations.

The LMC consists of an outer body that appears elliptical in projection on the sky, with a pronounced, off-center bar. The appearance in the optical wavelength regime is dominated by regions of strong star formation, and patchy dust absorption. However, when only RGB and carbon stars are selected from near-IR surveys such as 2MASS, the appearance of the LMC morphology is actually quite regular and smooth, apart from the central bar. Van der Marel (2001) found that at radii $r \gtrsim 4^{\circ}$ the contour shapes converge to an approximately constant position angle $\mathrm{PA}_{\mathrm{maj}}=189.3^{\circ} \pm 1.4^{\circ}$ and ellipticity $\epsilon=0.199 \pm 0.008$. A disk that is intrinsically circular will appear elliptical in projection on the sky, with the major axis position angle $\mathrm{PA}_{\mathrm{maj}}$ of the projected body equal to the line-of-nodes position angle $\Theta$. The fact that for the LMC $\Theta \neq \mathrm{PA}_{\text {maj }}$ implies that the LMC cannot be intrinsically circular. When the LMC viewing angles are used to deproject the observed morphology, this yields an in-plane ellipticity $\epsilon$ in the range $\sim 0.2-0.3$. This is larger than typical for disk galaxies, and is probably due to tidal interactions with either the SMC or the Milky Way.

\subsection{Transverse motion and kinematical distance}

As discussed in Section 2.1, the line-of-sight velocity field constrains the value of $w_{t s}=$ $v_{t s}+D_{0}(d i / d t)$. The carbon star analysis in vdM02 yields $w_{t s}=-402.9 \pm 13.0 \mathrm{~km} \mathrm{~s}^{-1}$. With the assumptions of a known LMC distance $D_{0}=50.1 \pm 2.5 \mathrm{kpc}$ (based on the distance modulus $m-M=18.50 \pm 0.10$ adopted by Freedman et al. (2001) on the basis of a review of all published work) and a constant inclination angle with time $(d i / d t=0)$ this yields an estimate of one component of the LMC transverse velocity. Some weaker constraints can also be obtained for the second component. The resulting region in LMC transverse velocity space implied by the carbon star velocity field is shown in Figure 8 of vdM02. This region is entirely consistent with the Hubble Space Telescope (HST) proper motion determination discussed in Section 4.1 below, and therefore provides an important consistency check on the latter. Alternatively, one can use the HST proper motion determination with the measured $w_{t s}$ and the assumption that $d i / d t=0$ to obtain a kinematic distance estimate for the LMC. This yields $m-M=18.57 \pm 0.11$, quite consistent with the Freedman et al. (2001) value.

Previous proper motion estimates for the LMC were lower than the current HST measurements. This introduced artifacts in previous analyses of the internal LMC velocity field (which ultimately depends on subtraction of the $v_{t}$ term in eq. [2.1] from the observed line-of-sight velocity field). For example, the H I velocity field of the LMC presented by Kim et al. (1998) showed a pronounced S-shape in the zero-velocity contour. Olsen \& 
Massey recently showed that this S-shape straightens out when the LMC HST proper motion measurement is used instead. So this too provides an independent consistency check on the validity of the HST proper motion measurement.

\subsection{Rotation curve and mass}

The rotation curve of the LMC rises approximately linearly to $R^{\prime} \approx 4 \mathrm{kpc}$, and stays roughly flat at a value $V_{\text {rot }}$ beyond that. The carbon star analysis of vdM02 with the HST proper motion measurement yields $V_{\text {rot }}=61 \mathrm{~km} \mathrm{~s}^{-1}$. By contrast, for H I one obtains $V_{\text {rot }}=80 \mathrm{~km} \mathrm{~s}^{-1}$ and for red supergiants $V_{\text {rot }}=107 \mathrm{~km} \mathrm{~s}^{-1}$ (Olsen \& Massey 2007). The random errors on these numbers are only a few $\mathrm{km} / \mathrm{s}$ in each case, due to the large numbers of independent velocity samples. Piatek, Pryor \& Olszewski (2008; hereafter P08) recently argued for an even higher $V_{\text {rot }}=120 \pm 15 \mathrm{~km} \mathrm{~s}^{-1}$ based on rotation measurements in the plane of the sky (based on the same proper motion observations discussed in Section 4.1 below). All of these measurements are influenced by uncertainties in the LMC inclination. However, the uncertainties have different sign for the $V_{\text {rot }}$ values inferred from line-of-sight velocities than and for those inferred from proper motions. The $V_{\text {rot }}$ measurement of P08 becomes more consistent with the line-of-sight measurements if the inclination is lower than the canonical values quoted in Section 2.3. The $V_{\text {rot }}$ estimates from line-of-sight velocities all have an additional uncertainty due to uncertainties in the LMC transverse motion. In the end, all $V_{\text {rot }}$ estimates therefore have a systematic error of $\sim 10 \mathrm{~km} \mathrm{~s}^{-1}$.

The differences between the $V_{\text {rot }}$ estimates for various tracers are significant, and cannot be attributed to either random or systematic errors. The fact that the $\mathrm{H}$ I gas rotates faster than the carbon stars can probably be largely explained as a result of asymmetric drift, with the velocity dispersion of the carbon stars being higher than that of the $\mathrm{H}$ I gas (see Section 2.6 below). The difference between the rotation velocities of $\mathrm{H}$ I and red supergiants is more puzzling, and may point towards non-equilibrium dynamics. There are in fact clear disturbances in the kinematics of the various tracers (Olsen \& Massey 2007). Moreover, the dynamical center of the $\mathrm{H}$ I is offset by $\sim 1 \mathrm{kpc}$ from the dynamical and photometric center of the stars (see, e.g., Cole et al. (2005) for a visual representation of the various relevant centroids of the LMC). All this complicates the inference of the underlying circular velocity of the gravitational potential.

If we use the $V_{\text {rot }}$ of the H I as a proxy for the circular velocity, and use the fact that the carbon star rotation curve remains flat out to the outermost datapoint at $\sim 9 \mathrm{kpc}$, then the implied LMC mass is $M_{\mathrm{LMC}}(9 \mathrm{kpc})=(1.3 \pm 0.3) \times 10^{10} \mathrm{M}_{\odot}$. The mass will continue to rise linearly beyond that radius for as long as the rotation curve remains flat. By contrast, the total stellar mass of the LMC disk is $\sim 2.7 \times 10^{9} \mathrm{M}_{\odot}$ and the mass of the neutral gas in the LMC is $\sim 0.5 \times 10^{9} \mathrm{M}_{\odot}$ (Kim et al. 1998). The combined mass of the visible material in the LMC is therefore insufficient to explain the dynamically inferred mass, and the LMC must be embedded in a dark halo.

\subsection{Velocity dispersion and vertical structure}

As in the Milky Way, younger populations have a smaller velocity dispersion (and hence a smaller scale height) than older populations. Measurements, in order of increasing dispersion, include: $\sim 9 \mathrm{~km} \mathrm{~s}^{-1}$ for red supergiants (Olsen \& Massey 2007); $16 \mathrm{~km} \mathrm{~s}^{-1}$ for H I gas (Kim et al. 1998); $\sim 20 \mathrm{~km} \mathrm{~s}^{-1}$ for carbon stars (vdM02); $\sim 25 \mathrm{~km} \mathrm{~s}^{-1}$ for RGB stars (Zhao et al. 2003; Cole et al. 2005); $\sim 30 \mathrm{~km} \mathrm{~s}^{-1}$ for star clusters (Schommer et al. 1992; Grocholski et al. 2006); $33 \mathrm{~km} \mathrm{~s}^{-1}$ for old long-period variables (Bessell, Freeman \& Wood 1986); $40 \mathrm{~km} \mathrm{~s}^{-1}$ for the lowest metallicity red giant branch stars with $[\mathrm{Fe} / \mathrm{H}]<-1.15$ (Cole et al. 2005); and $\sim 50 \mathrm{~km} \mathrm{~s}^{-1}$ for RR Lyrae stars (Minniti et al. 2003; Borissova et al. 2006). 
For the stars with the highest dispersions it has been suggested that they may form a halo distribution, and not be part of the LMC disk. On the other hand, this remains unclear, since the kinematics of these stars have typically been observed only in the central region of the LMC. Therefore, it is not known whether the rotation properties of these populations are consistent with being a separate halo component. In fact, the surface density distribution of the LMC RR Lyrae stars is well fit by an exponential with the same scale length as inferred for other tracers known to reside in the disk (Alves 2004). Either way, the vertical extent of all LMC populations is certainly significant. For example, even the (intermediate-age) carbon stars only have $V / \sigma \approx 3$. For comparison, the thin disk of the Milky Way has $V / \sigma \approx 9.8$ and its thick disk has $V / \sigma \approx 3.9$.

The velocity residuals with respect to a rotating disk model do not necessarily follow a Gaussian distribution. Although Zhao et al. (2003) did not find large deviations from a Gaussian for RGB stars, Graff et al. (2000) found that the carbon star residuals are better fit by a sum of Gaussians. More recently, Olsen \& Massey (2007) showed that some fraction of both carbon stars and red supergiants have peculiar kinematics that suggest an association with tidally disturbed features previously identified in H I.

\section{SMC kinematics}

The SMC structure and kinematics are less well studied and understood than those of the LMC. The morphological appearance in blue optical light is patchy and irregular. Kinematical observations of $\mathrm{H}$ I and young stars reveal ordered rotation that indicates that these tracers may reside in a disk. However, detailed velocity field fits using equation (2.1) have not been attempted. Stanimirović, Staveley-Smith \& Jones (2004) found that the H I rotation curve in the SMC rises almost linearly to $V_{\text {rot }} \approx 50 \mathrm{~km} \mathrm{~s}^{-1}$ at the outermost datapoint $(\sim 3.5 \mathrm{kpc})$, with no signs of flattening. The implied dynamical mass inside this radius is $2.4 \times 10^{9} \mathrm{M}_{\odot}$. By contrast, the total stellar mass of the SMC is $\sim 3.1 \times 10^{8} \mathrm{M}_{\odot}$ and the mass of the neutral gas is $5.6 \times 10^{8} \mathrm{M}_{\odot}$. The combined mass of the visible material in the SMC is therefore insufficient to explain the dynamically inferred mass, and the SMC must be embedded in a dark halo. $\dagger$

Evans \& Howarth (2008) obtained velocities for 2045 young (O, B, A) stars in the SMC, and found a velocity gradient of similar slope as seen in the H I gas. Surprisingly though, they find a position angle for the line of maximum velocity gradient that is quite different - almost orthogonal to that seen in H I. This may be an artifact of the different spatial coverage of the two studies (Evans \& Howarth did not observe in the North-East region where H I velocities are largest), since it would be hard to find a physical explanation for a significant difference in kinematics between $\mathrm{H}$ I gas and young stars.

When the old red stars that trace most of the stellar mass are isolated using CMDs, the morphological appearance of the SMC is more spheroidal (Zaritsky et al. 2000, 2002; Cioni, Habing \& Israel 2000; Maragoudaki et al. 2001). Harris \& Zaritsky (2006) studied the kinematics of 2046 RGB stars and inferred a velocity dispersion $\sigma=27.5 \pm 0.5 \mathrm{~km} \mathrm{~s}^{-1}$. This is similar to the dispersion of the young stars observed by Evans \& Howarth (2008), but unlike the young stars, the older RGB stars do not show much rotation. Their low $V_{\text {rot }} / \sigma$ is consistent with what is typical for $\mathrm{dE}$ and $\mathrm{dSph}$ galaxies. Hence, the SMC may be more akin to those galaxy types than to other more irregular systems.

Studies of the distances of individual tracers in the SMC have shown it to be much more vertically extended than would be expected for a disk galaxy. Crowl et al. (2001)

$\dagger$ These values are based on the analysis in Stanimirović et al. (2004), although those authors do not draw the same conclusion. 
mapped the distances of star clusters using red clump magnitudes. They argued that the SMC has axial ratios of 1:2:4, and is viewed almost pole on. While different authors have found a range of other axial ratios using different types of tracers, most authors agree that the SMC has a considerable line-of-sight depth.

\section{Orbital history of the Magellanic Clouds}

To understand the history of the Magellanic System and the origin of the Magellanic Stream it is important to know the orbit of the Magellanic Clouds around the Milky Way. This requires for each Cloud knowledge of all three of the velocity components of the center of mass. Line-of-sight velocities can be accurately determined from the Doppler velocities of tracers. However, determination of the velocity in the plane of the sky through proper motions is much more difficult. This was the primary obstacle for a long time, but recent breakthroughs have now yielded considerable progress.

\subsection{Proper motions}

Previous attempts at measuring the proper motions (PMs) of the LMC and SMC from the ground or with Hipparcos were reviewed in vdM02. However, this earlier work has now been largely superseded by the studies performed with the ACS/HRC on HST by Kallivayalil et al. (2006a,b; hereafter K06a,b). Two epochs of data were obtained with a $\sim 2$ year time baseline for 21 fields in the LMC and 5 fields in the SMC, all centered on background quasars identified from the MACHO database (Geha et al. 2003). PMs were obtained for each field by measuring the average shift of the stars with respect to the background quasar. Upon correction for the orientation and rotation of the LMC disk, each field yields an independent estimate of the center-of-mass PM. The average for the different fields yields the final estimate, while the RMS among the results from the $N$ different fields yields the PM error RMS $/ \sqrt{N}$.

K06a,b obtained for the PMs in the West and East directions that

$$
\begin{array}{ll}
\mu_{W}=-2.03 \pm 0.08 \operatorname{mas~yr}^{-1}, & \mu_{N}=0.44 \pm 0.05 \mathrm{mas} \mathrm{yr}^{-1} \\
\mu_{W}=-1.16 \pm 0.18 \mathrm{mas} \mathrm{yr}^{-1}, & \mu_{N}=-1.17 \pm 0.18 \mathrm{mas} \mathrm{yr}^{-1}
\end{array}
$$

The same data were reanalyzed more recently by P08. Using an independent analysis with different software and point spread function models they obtained that

$$
\begin{aligned}
& \mu_{W}=-1.96 \pm 0.04 \operatorname{mas~yr}^{-1}, \quad \mu_{N}=0.44 \pm 0.04 \text { mas yr}^{-1} \\
& \mu_{W}=-0.75 \pm 0.06 \operatorname{mas~yr}^{-1}, \quad \mu_{N}=-1.25 \pm 0.06 \text { mas yr}^{-1} \quad(S M C) \text {. }
\end{aligned}
$$

P08 made magnitude-dependent corrections for small charge transfer inefficiency effects. By contrast, K06a,b assumed that these effects (always along the detector y axis) average to zero over all fields because of the random telescope orientations used for different fields. The fact the the results from these two studies are generally in good agreement for the LMC confirms the validity of this assumption. However, the explicit correction applied by P08 does yield better agreement between different fields, and therefore smaller errorbars. For the SMC, the results for the individual fields are in good agreement between the studies. However, the studies used different methods for weighted averaging of the fields, with K06a,b being more conservative and allowing for potential unknown systematic effects. This produces larger errorbars than in P08 and a significant difference in $\mu_{W}$.

Transformation of the PMs to a space velocity in $\mathrm{km} \mathrm{s}^{-1}$ requires knowledge of the distance $D_{0}$. For the LMC, $D_{0} \approx 50.1 \mathrm{kpc}$, so that 1 mas yr${ }^{-1}$ corresponds to $238 \mathrm{~km} \mathrm{~s}^{-1}$. For the SMC, $D_{0} \approx 61.6 \mathrm{kpc}$, so that $1 \mathrm{mas} \mathrm{yr}^{-1}$ corresponds to $293 \mathrm{~km} \mathrm{~s}^{-1}$. After transformation of the PM to $\mathrm{kms}^{-1}$, it can be combined with the observed center-of-mass 
line-of-sight velocity to obtain the full three-dimensional velocity vector. For the LMC, $v_{\mathrm{sys}}=262.2 \pm 3.4 \mathrm{~km} \mathrm{~s}^{-1}(\mathrm{vdM} 02)$; and for the SMC, $v_{\mathrm{sys}}=146 \pm 0.6 \mathrm{~km} \mathrm{~s}^{-1}$ (Harris $\&$ Zaritsky 2006). The resulting vectors can be corrected for the solar reflex motion and transformed to the Galactocentric rest-frame as described in vdM02. For the LMC this yields that the motion has a radial component of $V_{\text {rad }}=89 \pm 4 \mathrm{~km} \mathrm{~s}^{-1}$ pointing away from the Galactic center, and a tangential component of $V_{\tan }=367 \pm 18 \mathrm{~km} \mathrm{~s}^{-1} . \dagger$

\subsection{Orbit around the Milky Way}

The combination of a small but positive radial velocity and a tangential velocity that exceeds the circular velocity of the Milky Way halo implies that the Clouds must be just past pericenter. The calculation of an actual orbit requires detailed knowledge of the gravitational potential of the Milky Way dark halo. Past work had generally assumed that the dark halo can be approximated by a spherical logarithmic potential. Estimates of the transverse velocities of the Clouds based on models of the Magellanic Stream had suggested that for the LMC $V_{\text {tan }}=287 \mathrm{~km} \mathrm{~s}^{-1}$ (e.g., Gardiner, Sawa \& Fujimoto 1994; Gardiner \& Noguchi 1996). This then yielded an orbit with an apocenter to pericenter ratio of $\sim 2.6: 1$ and an orbital period of $\sim 1.6$ Gyr. This orbit was adopted by most subsequent modeling studies of the Magellanic System. However, the new HST PM measurements significantly revise this view. The observed $V_{\text {tan }}$ is $80 \pm 16 \mathrm{~km} \mathrm{~s}^{-1}$ larger than the Gardiner \& Noguchi (1996) value, and therefore inconsistent with it at $\sim 5 \sigma$. The observed value implies a much larger apocenter distance (in excess of $200 \mathrm{kpc}$ ) at which the assumption of a logarithmic potential is not a good assumption.

Motivated by these considerations, Besla et al. (2007) performed a new study of the Magellanic Cloud orbits using an improved Milky Way model, combined with the K06a,b HST PMs. The Milky Way model was chosen similar to that proposed in Klypin, Zhao \& Somerville (2002). It consists of disk, bulge, hot gaseous halo, and dark halo components. The dark halo has a $\Lambda$ CDM-motivated NFW potential with adiabatic contraction. In the fixed Milky Way potential, the orbits of the LMC and SMC were integrated backwards in time, starting from the current observed positions and velocities. The extent of the galaxies was taken into account in the calculation of their mutual gravitational interaction, and a parameterized prescription was used to account for dynamical friction. The gravitational influence of M31 can be taken into account in this formalism as well, but this make little difference to the results (Kallivayalil 2007; Shattow \& Loeb 2009).

The most favored Milky Way model presented by Klypin et al. (2002) has a total mass $M=10^{12} \mathrm{M}_{\odot}$. In this model, the escape velocity at $50 \mathrm{kpc}$ is $\sim 380 \mathrm{~km} \mathrm{~s}^{-1}$. This is very similar to the observed $V_{\mathrm{tan}}$ of the LMC, and as a result, the inferred orbit is approximately parabolic, with no previous pericenter passage. In other words, the Magellanic Clouds are passing by the Milky Way now for the first time. To obtain an orbit that is significantly bound, $\mu_{W}$ would have to be larger by $\sim+0.3$ mas yr$^{-1}$ ( $4 \sigma$ with the K06a errorbar, or $7 \sigma$ with the P08 errorbar). Alternatively, it is possible that the Milky Way is more massive (Smith et al. 2007; Shattow \& Loeb 2008). A mass of $M=2 \times 10^{12} \mathrm{M}_{\odot}$ is more or less the largest mass consistent with the available observational constraints (see also the discussion in van der Marel \& Guhathakurta 2008). This would produce a bound orbit. However, with either the larger Milky Way mass or with the larger $\mu_{W}$, the orbit would still be quite different than has been previously assumed in models of the Magellanic System. There would be only 1 previous pericenter passage, the apocenter distance would be $400 \mathrm{kpc}$ or more, and the period would be

$\dagger$ These velocities are based on the K06a PM values. However, use of the P08 PM values would yield similar velocities that would not alter the arguments in the remainder of the text. 
6-7 Gyr. $\ddagger$ Therefore, the new PM results drastically alter our view of the history of the Magellanic System.

The view that the Magellanic Clouds may be passing by the Milky Way for the first time may seem revolutionary at first. However, there are arguments to consider this reasonable. Van den Bergh (2006) pointed out that the LMC and SMC are unusual in that they are the only satellites in the Local Group that are both gas rich and located close to their parent galaxy. He suggested based on this that the Magellanic Clouds are interlopers that were originally formed in the outer reaches of the Local Group. Moreover, cosmological simulations show that: (a) accretion of LMC-sized subhalos by Milky-Way sized halos is common since $z \sim 1$; and (b) finding long-term satellites with small pericenter distances around Milky-Way sized halos is rare (Kazantzidis, Zentner \& Bullock 2008). Therefore, a scenario in which the Magellanic Clouds are passing by the Milky Way now for the first time seems more likely from a purely cosmological perspective than a scenario in which they have been satellites for many orbital periods of 1-2 Gyr each.

\section{3. $L M C-S M C$ orbit}

Although the Magellanic Clouds may not be bound to the Milky Way, it would be much more unlikely for the Magellanic Clouds not to be bound to each other. The likelihood of two satellite galaxies running into each other by chance is quite low. Also, various properties of the Clouds (such as their common H I envelope) suggest that they have been associated with each other for a significant time. The K06a,b PMs imply a relative velocity between the SMC and LMC of $105 \pm 42 \mathrm{~km} \mathrm{~s}^{-1}$. Orbit calculations (K06b; Besla et al. 2007) show that the error bar on this is too large to say with any confidence whether or not they are indeed bound. However, binary orbits do exist within the $1 \sigma$ error ellipse, so there seems little reason to depart from this null hypothesis. Indeed, there are allowed orbits that have close passages between the Clouds at $\sim 0.3 \mathrm{Gyr}$ and $\sim 1.5 \mathrm{Gyr}$ in the past (Besla et al., these proceedings). These are the time scales that have been previously associated with the formation of the Magellanic Bridge and Stream, respectively.

\subsection{Magellanic Stream}

The Magellanic Stream is discussed in detail in other contributions in this volume. One important thing to note though in the present context is that the new insights into the orbit of the Magellanic Clouds around the Milky Way drastically affect our understanding of the Magellanic Stream. The Stream does not lie along the projected path on the sky traced by the LMC and SMC orbits, and the HI velocity along the Stream is not as steep as that along the orbits (Besla et al. 2007). This is inconsistent with purely tidal models of the stream (e.g., Gardiner \& Noguchi 1996; Connors, Kawata \& Gibson 2006). Moreover, the more limited number of passages through the Milky Way disk, and the larger radius at which this occurs, imply that the ram pressure models that have been proposed (e.g., Moore \& Davis 1994; Mastropietro et al. 2005) probably won't work either. It is therefore essential that models of the Magellanic Stream be revisited, with an eye towards inclusion of new physics and exploration of new scenarios (see Besla et al., these proceedings). The recent finding by Nidever, Majewski \& Burton (2008) that one filament of the Magellanic Stream, containing more than half its gas mass, can be traced back to the 30 Doradus star forming region in the LMC is particularly interesting in this context. This indicates that an outflow may have created or contributed to the Stream, which has not been addressed in previous models.

$\ddagger$ if this were the case, then the mass build-up of the Milky Way with time, as in, e.g., Wechsler et al. (2002), would also have to be taken into account for calculation of an accurate orbit. 


\section{Concluding remarks}

The kinematics of the LMC are now fairly well understood, with velocities of thousands of individual tracers of various types having been fitted in considerable detail with (thick) disk models. Questions that remain open for further study include the reality and origin of kinematical differences between different stellar tracer populations, the differences between the gaseous and stellar kinematics, and the amount and origin of non-equilibrium features in the kinematics. The kinematics of the SMC are understood more poorly, but appear generally consistent with being a spheroidal system of old stars with an embedded irregular disk of gas and young stars.

The HST PM work has provided the most surprising results in recent years, with important implications for both the history of the Magellanic System and the origin of the Magellanic Stream. Of course, it is natural in discussions about this to wonder about the robustness of the observational results. It should be noted in this context that many experimental features and consistency checks are built in that support the general validity of the HST PM results. These include: (1) the use of random telescope orientations causes systematic errors tied to the detector frame to cancel out when averaging over all fields; (2) the final PM errors are based on the observed scatter between fields, with no assumptions about the source and nature of the underlying errors; (3) two groups used different methods to analyze the same data and obtained consistent results; (4) P08 managed to measure a PM rotation curve for the LMC that is broadly consistent with expectation, which would have been impossible if the PM errors were in reality larger than claimed; (5) the difference between the LMC and SMC PMs is more or less consistent with expectation for a binary orbit, which would not generally have been the case if the measurements suffer from unknown systematics; (6) the LMC PM is consistent with expectation based on the line-of-sight velocity field of carbon stars (see Section 2.4); and (7) the LMC PM leads to an H I velocity field with a straight zero-velocity curve, by contrast to previously assumed values (see Section 2.4).

One interesting feature in the observational PM results is that with the P08 PM values, there are no bound LMC-SMC orbits, given their different $\mu_{W}$ and smaller error bars for the SMC compared to the K06b results. However, the SMC PM is significantly less certain that that for the LMC, due to the smaller number of fields observed with $H S T$, and the fact that most of them were observed at a similar telescope orientation (which implies that potential systematic errors that are fixed in the detector frame do not average out when the results from different fields are combined). This underscores the need for additional PM observations. A third epoch of observations for most fields has already been obtained with $H S T /$ WFPC2, and preliminary analysis supports the validity of the results based on the first two epochs (Kallivayalil et al., these proceedings). A fourth epoch is planned with $H S T / \mathrm{ACS}$ and $H S T / \mathrm{WFC} 3$ in 2009. With the increased time baselines and use of multiple different instruments it will be possible to further reduce random errors and constrain possible systematic errors. In turn, this will allow new scientific problems to be addressed, such as the internal proper motion kinematics of the Clouds, and their rotational parallax distances (the distances obtained by equating the line-of-sight and proper motion rotation curves).

\section{References}

Alves, D. R. 2004, ApJ, 601, L151

Besla, G., Kallivayalil, N, Hernquist, L., Robertson, B., Cox, T. J., van der Marel, R. P., \& Alcock, C. 2007, ApJ, 668, 949

Bessell, M. S., Freeman, K. C., \& Wood, P. R. 1986, ApJ, 310, 710 
Borissova, J., Minniti, D., Rejkuba, M., \& Alves, D. 2006, A\&SA, 460, 459

Cioni, M. -R. L., Habing, H. J., \& Israel, F. P. 2000, A\&A, 358, L9

Cole, A. A., Tolstoy, E., Gallagher, J. S., III, \& Smecker-Hane, T. A. 2005, AJ, 129, 1465

Connors, T. W., Kawata, D., \& Gibson, B. K. 2006, MNRAS, 371, 108

Crowl, H. H., Sarajedini, A., Piatti, A. E., Geisler, D., Bica, E., Claria, J. J., \& Santos, J. F. C., Jr. 2001, AJ, 122, 220

Evans, C. J. \& Howarth, I. D. 2008, MNRAS, 386, 826

Feitzinger, J. V., Schmidt-Kaler, T., \& Isserstedt, J. 1977, $A \mathscr{E} A, 57,265$

Freedman, W. L., Madore, B. F., Gibson, B. K., et al. 2001, ApJ, 553, 47

Gardiner, L. T. \& Noguchi, M. 1996, MNRAS, 278, 191

Gardiner, L. T., Sawa, T., \& Fujimoto, M. 1994, MNRAS, 266, 567

Geha, M., Alcock, C., Allsman, R. A., et al. 2003, AJ, 125, 1

Graff, D. S., Gould, A. P., Suntzeff, N. B., Schommer, R. A., \& Hardy, E. 2000, ApJ, 540, 211

Grocholski, A. J., Cole, A. A., Sarajedini, A., Geisler, D., \& Smith, V. V. 2006, AJ, 132, 1630

Harris, J. \& Zaritsky, D. 2006, AJ, 131, 2514

Kallivayalil, N., van der Marel, R. P., Alcock, C., Axelrod, T., Cook, K. H., Drake, A. J., \& Geha, M. 2006a, ApJ, 638, 772 (K06a)

Kallivayalil, N., van der Marel, R. P., \& Alcock, C. 2006b, ApJ, 652, 1213 (K06b)

Kallivayalil, N. 2007, PhD thesis, Harvard University

Kazantzidis, S., Zentner, A. R., \& Bullock, J. S. 2008, ApJ, in press [arXiv:0807.2863]

Kim, S., Staveley-Smith, L., Dopita, M. A., Freeman, K. C., Sault, R. J., Kesteven M. J., \& McConnell, D. 1998, ApJ, 503, 674

Klypin, A., Zhao., H. S., \& Somerville, R. S. 2002, ApJ, 573, 597

Kunkel, W. E., Irwin, M. J., \& Demers, S. 1997, A\&AS, 122, 463

Maragoudaki, F., Kontizas, M., Morgan, D. H., Kontizas, E., Dapergolas, A., \& Livanou, E. 2001, A\&A 379,864

Mastropietro, C., Moore, B., Mayer, L., Wadsley, J., \& Stadel, J. 2005, MNRAS, 363, 509

Meatheringham, S. J., Dopita, M. A., Ford, H. C., \& Webster, B. L. 1988, ApJ, 327, 651

Minniti, D., Borissova, J., Rejkuba, M., Alves, D. R., Cook, K. H., \& Freeman, K. C. 2003, Science, 301, 1508

Moore, B. \& Davis, M. 1994, MNRAS, 270, 209

Nidever, D. L., Majewski, S. R., \& Burton, W. B. 2008, ApJ, 679, 432

Nikolaev, S., Drake, A. J., Keller, S. C., Cook, K. H., Dalal, N., Griest, K., Welch, D. L., \& Kanbur, S. M. 2004, ApJ, 601, 260

Olsen, K. A. G. \& Salyk, C. 2002, AJ, 124, 2045

Olsen, K. A. G. \& Massey, P. 2007, ApJ, 656, L61O

Persson, S. E., Madore, B. F., Krzeminski, W., Freedman, W. L., Roth, M., \& Murphy, D. C. 2004, AJ, 128, 2239

Piatek, S., Pryor, C., \& Olszewski, E. W. 2008, ApJ, 135, 1024 (P08)

Schommer, R. A., Suntzeff, N. B., Olszewski, E. W., \& Harris, H. C. 1992, AJ, 103, 447

Shattow, G. \& Loeb, A. 2009, MNRAS, 392, L21

Smith, M. C., Ruchti, G. R., Helmi, A., et al. 2007, MNRAS, 379, 755

Stanimirović, S., Staveley-Smith, L., \& Jones, P. 2004, ApJ, 604, 176

Subramaniam, A. 2003, ApJ, 598, L19

van den Bergh, S. 2006, $A J, 132,1571$

van der Marel, R. P. \& Cioni, M.-R. 2001, AJ, 122, 1807

van der Marel, R. P. 2001, AJ, 122, 1827

van der Marel, R. P., Alves, D. R., Hardy, E., \& Suntzeff, N. B. 2002, AJ, 124, 2639 (vdM02)

van der Marel, R. P. \& Guhathakurta, P. 2008, ApJ, 678, 187

Wechsler, R. H., Bullock, J. S., Primack, J. R., Kravtsov, A. V., \& Dekel, A. 2002, ApJ, 568, 52

Weinberg, M. D. 2000, ApJ, 532, 922

Zaritsky, D., Harris, J., Grebel, E. K., \& Thompson, I. B. 2000, ApJ, 534, L53

Zaritsky, D., Harris, J., Thompson, I. B., Grebel, E. K., \& Massey, P. 2002, AJ, 123, 855

Zhao, H., Ibata, R. A., Lewis, G. F., \& Irwin, M. J. 2003, MNRAS, 339, 701 